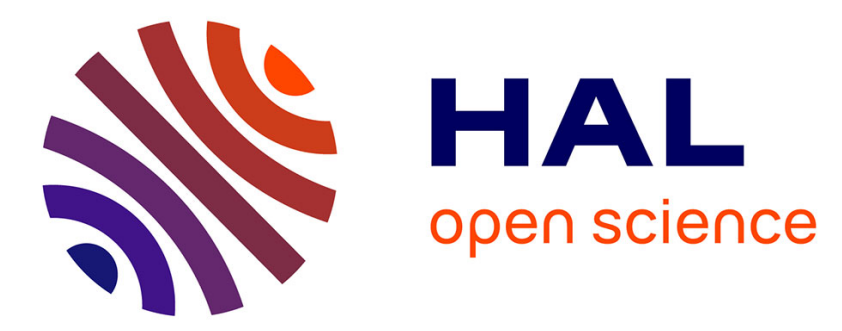

\title{
Coexistence of an ordered anisotropic phase and a liquid expanded phase in an amphiphilic monolayer
}

\author{
H. Bercegol, F. Gallet, D. Langevin, J. Meunier
}

\section{To cite this version:}

H. Bercegol, F. Gallet, D. Langevin, J. Meunier. Coexistence of an ordered anisotropic phase and a liquid expanded phase in an amphiphilic monolayer. Journal de Physique, 1989, 50 (16), pp.2277-2289. 10.1051/jphys:0198900500160227700 . jpa-00211059

\section{HAL Id: jpa-00211059 https://hal.science/jpa-00211059}

Submitted on 1 Jan 1989

HAL is a multi-disciplinary open access archive for the deposit and dissemination of scientific research documents, whether they are published or not. The documents may come from teaching and research institutions in France or abroad, or from public or private research centers.
L'archive ouverte pluridisciplinaire HAL, est destinée au dépôt et à la diffusion de documents scientifiques de niveau recherche, publiés ou non, émanant des établissements d'enseignement et de recherche français ou étrangers, des laboratoires publics ou privés. 
Classification

Physics Abstracts

$64.60-68.10-78.20$

\title{
Coexistence of an ordered anisotropic phase and a liquid expanded phase in an amphiphilic monolayer
}

\author{
H. Bercegol, F. Gallet, D. Langevin and J. Meunier \\ Laboratoire de Physique Statistique de l'ENS, 24 rue Lhomond, 75231 Paris Cedex 05, France
}

(Reçu le 8 février 1989, accepté le 3 mai 1989)

\begin{abstract}
Résumé. - Nous présentons une étude expérimentale d'une transition de phase d'une monocouche d'amphiphiles fluorescents. Nous avons effectué des mesures de pression de surface, d'ellipsométrie et des observations en microscopie. Nous avons observé une phase ordonnée anisotrope en coexistence avec une phase liquide. La phase ordonnée est constituée de domaines allongés dont la croissance et la fusion sont décrites brièvement.

Abstract. - We present an experimental study of a phase transition in a monolayer made of fluorescent amphiphiles. We have combined measurement of surface tension isotherms, ellipsometry and observations by microscopy. We have observed an ordered anisotropic phase coexisting with a liquid phase. The ordered phase is made of elongated domains, whose growth and melting are briefly described.
\end{abstract}

\section{Introduction.}

Two-dimensional phase transitions in amphiphilic monolayers have been the subject of much experimental [1] and theoretical work [2]. However, even the simple problem of the transition order for the so-called liquid expanded-liquid condensed transition remained unclear for a long time. In the coexistence region, the surface pressure $\Pi$ still varies with the area per molecule $A$, i.e. the relative amount of the coexisting phases. This was ascribed to a possible second order character of the transition. However, the surface pressure variation was observed to strongly depend on the amount of impurities : in very careful experiments on pentadecanoic acid, Pethica and coworkers were able to obtain flat $\Pi(A)$ curves in the coexistence region [3].

The issue was resolved recently when it became possible to observe coexisting domains of liquid expanded and liquid condensed phases with a fluorescence microscope. For this purpose, a fluorescent molecule is added to the amphiphilic layer. When its partitioning between the coexisting phases is very different, the contrast in the fluorescence images is sufficient to visualize the different domains [4-6]. One drawback of the method is that the fluorescent molecules act as impurities in the monolayer and may affect the transition. Recently, Möhwald et al. improved the image collecting process in order to reduce the fluorescent molecule concentration to a very low level $(0.1 \%)$ [7]. In this limit, their particular observations are independent of the impurity concentration. 
We took a different approach, studying a monolayer consisting of only fluorescent amphiphiles : fluorescent labeled stearic acid. We observed a liquid expanded-liquid condensed-like transition. However the domains in the condensed phase show solid-like behavior and their shape and their optical properties are strongly anisotropic. We also have performed surface pressure and ellipsometry studies to check the two-dimensional character of the transition.

Elongated but spiral shaped 2D domains were already observed in mixed phospholipidcholesterol monolayers [5]. It is the first time that straight elongated optically anisotropic domains are observed in a single component system. Up to date, only very few theoretical predictions have been made for domains shape. We will however try to relate our observations to the existing models.

In the following, we will first describe the materials and methods, then present the results and their discussion.

\section{Materials and methods.}

2.1 SAMPLE PREPARATION. - The amphiphilic molecule used is stearic acid labeled with a fluorescent group (nitrobenzoxadiazol : NBD) attached in the middle of the chain (Fig. 1). This compound is currently called NBD-stearic acid. It was purchased from MOLECULAR PROBE and used without further purification. The purity claimed is $>99 \%$. The spreading solvent is chloroform mixed with $0.5 \%$ ethanol (MERCK for analysis). The aqueous substrate is ultrapure water from a MILLIPORE milli- $Q$ system, in which $0.01 \mathrm{M}$ hydrochloric acid is added in order to avoid fatty acid solubilization.

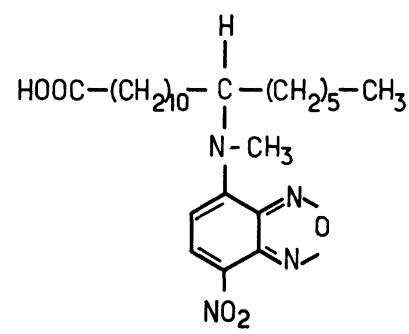

Fig. 1. - Chemical formula of the NBD-stearic acid.

2.2 EXPERIMENTAL TECHNIQUES. - The surface pressure measurements were made with a commercial Lauda film balance (at the Collège de France). The slowest compression rate in the concentration range investigated was $2 \AA^{2} /$ molecule/minute. Temperature was $(20 \pm 0.5)^{\circ} \mathrm{C}$.

Ellipsometry measurements were made with a home built apparatus using phase modulation technique [8]. When working at the Brewster angle, a light beam polarized at $45^{\circ}$ from the plane of incidence becomes elliptically polarized after reflection. The ellipticity $\bar{\rho}$ is the ratio of the reflected amplitudes for the field components polarized respectively parallel and perpendicular to the plane of incidence.

The ellipticity $\bar{\rho}$ has three different origins. The first one is the surface roughness ; in the presence of an adsorbed layer at the surface, this contribution to the ellipticity is negligible. The second one is the thickness of the monolayer. Assuming that the amphiphilic layer is homogeneous, of thickness $d$ and index of refraction $n_{\mathrm{f}}$, the ellipticity $\bar{\rho}^{\mathrm{L}}$ calculated from 
Drude's formula [9] is :

$$
\bar{\rho}^{\mathrm{L}}=\frac{\pi d}{\lambda} \sqrt{\frac{n_{\mathrm{w}}^{2}+1}{n_{\mathrm{w}}^{2}-1}} \frac{\left[n_{\mathrm{f}}^{2}-1\right]\left[n_{\mathrm{f}}^{2}-n_{\mathrm{w}}^{2}\right]}{n_{\mathrm{f}}^{2}}
$$

where $n_{\mathrm{w}}$ is the refractive index of water.

The third origin is the anisotropy of the refractive index in the monolayer. $\bar{\rho}$ can be deduced from light propagation laws in an anisotropic medium [10]. In the case of an optically uniaxial monolayer with the symmetry axis in the plane of the surface, $\bar{\rho}$ depends upon the angle of the axis with the incident plane. If the layer is made of randomly oriented uniaxial domains, the measured ellipticity $\bar{\rho}$ can be calculated by averaging over all the possible directions of the axis. One then finds :

$$
\bar{\rho}=\bar{\rho}^{\mathrm{L}}\left(n_{\mathrm{f}}=n_{\mathrm{fo}}\right)+\left(n_{\mathrm{fe}}^{2}-n_{\mathrm{fo}}^{2}\right) d / 2
$$

where $n_{\mathrm{fo}}$ and $n_{\mathrm{fe}}$ are respectively the ordinary and extraordinary refractive indices of the monolayer.

The fluorescence microscopy observations were made with a Reichert Polyvar Met microscope connected to a video camera Hamamatsu. Pictures of the TV screen are taken with a photocopy apparatus (Mitsubishi).

NBD stearic acid (in chloroform) has an absorption band centered at $480 \mathrm{~nm}$ and of approximate bandwidth $60 \mathrm{~nm}$. The corresponding absorption coefficient of a molar solution is $K_{\mathrm{M}}=5.3 \times 10^{4} \mathrm{~cm}^{-1}$, as determined with a spectrometer, roughly constant around $480 \mathrm{~nm}$. For the observations under microscope, we illuminate the NBD stearic acid monolayers with a $200 \mathrm{~W}$ mercury lamp through a band filter transmitting from 450 to $510 \mathrm{~nm}$. We observe the yellow fluorescence light through a long pass filter.

Both microscopy and ellipsometry are performed at room temperature $T=(21 \pm 1)^{\circ} \mathrm{C}$.

\section{Results.}

3.1 SURFACE PRESSURE EXPERIMENTS. - We show in figure 2 three isotherms corresponding to three different compression rate, respectively 22,8 and $4 \AA^{2} /$ molecule/minute. For areas $A$ larger than $110 \AA^{2} /$ molecule, our balance is not sensitive enough to get information about the liquid-gas coexistence transition. We will be interested here only in the behaviour at smaller areas.

In view of the compression rate dependence of the curves, we have determined an « equilibrium » curve, stopping the compression and waiting for each $A$ value until the surface pressure reaches a constant value. This takes about 30 minutes for each point. We have obtained in this way the curve of figure 3. The bumps of figure 2 , which mean negative compressibility and that are a clear indication of a non equilibrium compression, have disappeared as expected in figure 3. A break in slope occurs at the value $A_{\mathrm{c}}=84 \AA^{2}$, and the curve looks like the usual fatty acids and phospholipids monolayers isotherms in the region of the liquid expanded-liquid condensed phase transition; the large value of $A_{\mathrm{c}}$ reflects the bulkiness of the NBD side group on the chain. Below $A_{c}$, the surface pressure varies much more slowly upon compression. This pseudo-plateau indicates the occurrence of a phase transition. The bump shown in the curves of figure 2 strongly suggests that there is a nucleation barrier for the formation of the condensed phase and that the transition is first order. More evidence for the first order character can be found in the compression rate dependence of the curves, also observed upon decompression (Fig. 3). Note that the 


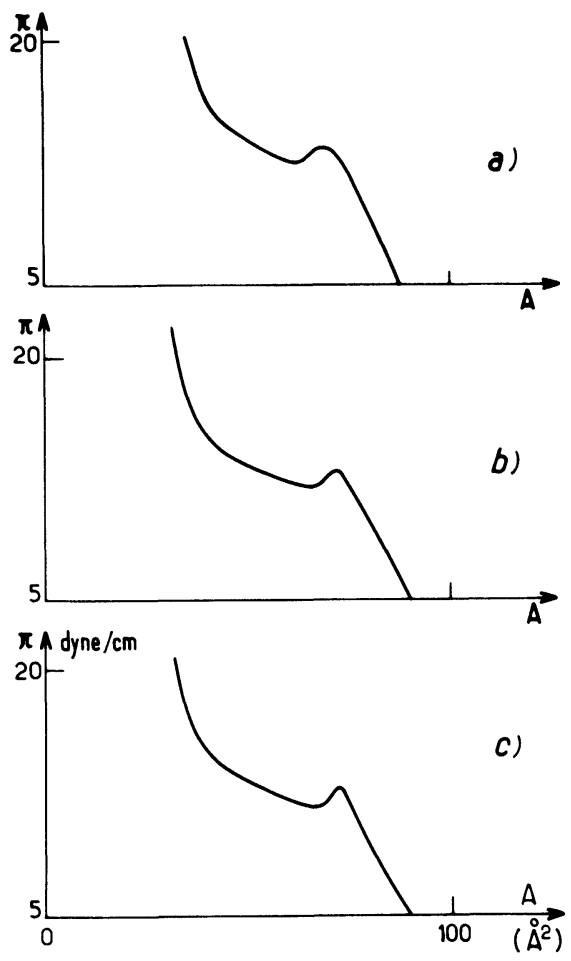

Fig. 2. - Surface pressure curves for different compression rates : a) $22 \AA^{2} /$ molecule/minute ; b) $8 \AA^{2} / \mathrm{mol} . / \mathrm{min}$. ; c) $4 \AA^{2} / \mathrm{mol} . / \mathrm{min}$.

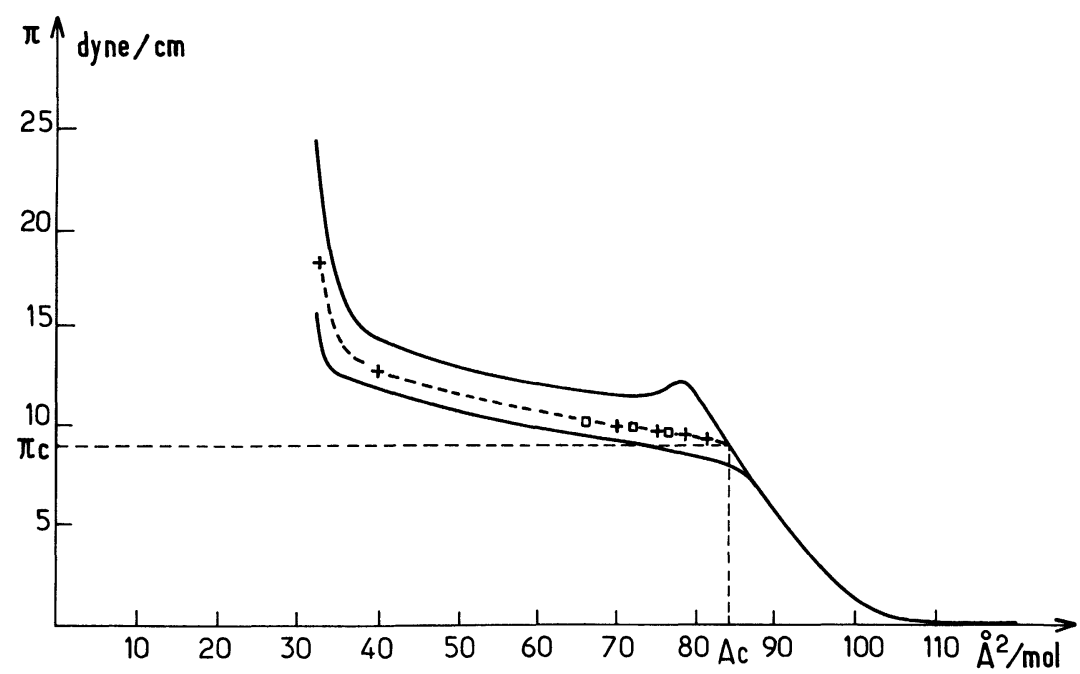

Fig. 3. - Surface pressure curves ; upper curve : continuous compression $\left(2 \AA^{2} / \mathrm{mol} . / \mathrm{min}\right.$. $)$; lower curve : continuous decompression; dashed curve : points obtained after waiting about 30 minutes (see text). The two different types of points correspond to two different monolayers : this illustrate the good reproducibility of the measurements. 
equilibrium measurements of the surface pressure were identical whether made by compression or decompression, and independent of the number of compressions performed, within experimental accuracy.

Below $25 \mathrm{~A}^{2} /$ molecule, the reproducibility disappears. The pressure only increases if $A$ is decreased rapidly. At low compression rates, pressure drops are observed. These features are characteristic of monolayer collapse. The pressure drops down to about $29 \mathrm{dyne} / \mathrm{cm}$, approximately the value of the equilibrium spreading pressure.

3.2 FluORESCENCE MICROSCOPY. - The microscope field is black when looking at the pure aqueous substrate. When the monolayer is spread in the liquid state, the field becomes weakly uniformly fluorescent.

When compressing the layer to a value $A$ slighly less than $A_{\mathrm{c}}=84 \AA^{2}$, small bright points appear after several minutes. Elongated, needle-like structures progressively form and grow around each point. When they stop growing, they all approximately have the same shape and length (Fig. 4a). The number of seeds increases with the initial departure from equilibrium, i.e. with $A-A_{\text {c }}$. A black line dividing the needles into two parts is sometimes observed.

The observed contrast between the solid domains and the liquid ones in fluorescence microscopy is very large and cannot be explained by different probe concentrations in the two phases. This suggests that the NBD-stearic acid molecule is bent toward the liquid phase. In this case, the NBD group of the molecule act as a second polar head whose fluorescence is quenched by the contact with water. At the liquid-solid transition, the molecule straightens up and the NBD group in this new configuration is far from water. This picture is supported by the large $A$ values at the liquid-solid transition.

When the monolayer is further compressed, the needles grow, apparently keeping the initial elongation ratio constant. If the compression is slow enough, their number stays constant (equal to the number of initial bright spots). A complete series of photos is shown in figures $4 \mathrm{a}-4 \mathrm{e}$. All the photos are taken when the pressure has reached its equilibrium value.

The needles tend to split at their tips, following the dark middle line (Fig. 4b). Structures looking like associations of single crystals growing along different direction axes can be observed (Figs. 4c-d).

When $A$ further decreases, two needles can collide, and their tips can break (Fig. 4e). As long as needles do not collide, their elongation ratio seems to be conserved.

At maximum compression, the microscope field is almost entirely filled by needles, but never totally ; figure 5 shows its appearance after waiting 30 minutes. The absence of merging of the condensed phase has also been observed in other systems [7, 11]. It is not yet fully understood.

If we now start increasing $A$, a slightly different behaviour is observed. The initial dark lines become wider. The needles split into parts before the complete melting. The whole process is fully reversible as soon as compression is started again from above $A_{\mathrm{c}}$.

3.3 ELLIPSOMETRY. - The surface pressure curves and the fluorescence microscopy suggest that there is a first order phase transition in the monolayer, between a liquid phase and an anisotropic two-dimensional phase. This is confirmed by ellipsometric measurements. These ones also show the existence of the usual gas-liquid transition.

Just above $110 \AA^{2} /$ molecule, the measured values of the ellipticity $\bar{\rho}$ fluctuate around a mean value (Fig. 6). This corresponds to the gas-liquid coexistence region. The same phenomenon occurs between 32 and $84 \AA^{2} /$ molecule, i.e. in the liquid-solid phase coexistence region. The amplitude of the fluctuations is very large, especially in the gas phase (up to $100 \%$ ). Similar results on pentadecanoic acid were recently reported by Rasing et al. [11]. The fluctuations are produced because the ratio of the area occupied by the two phases in the 


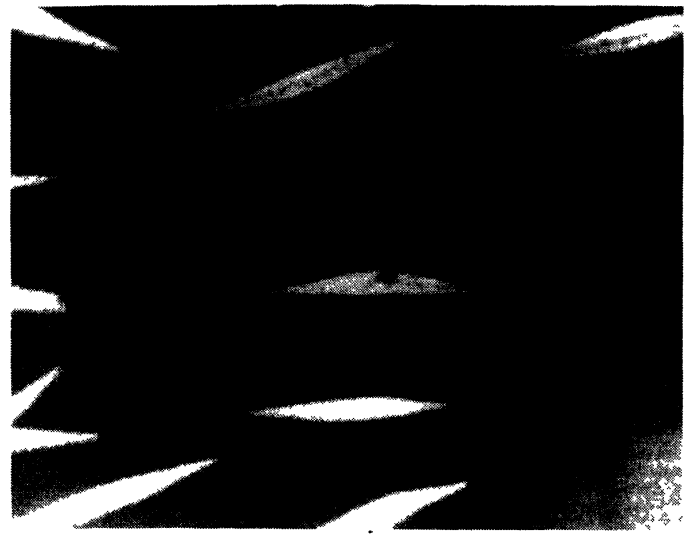

a)

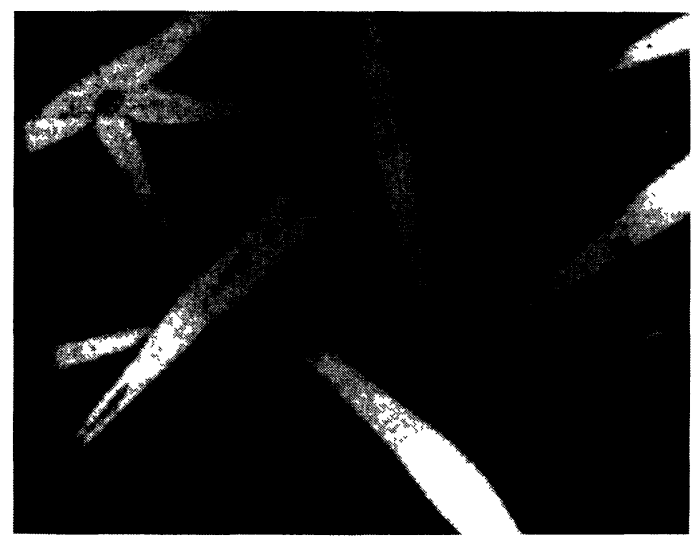

c)

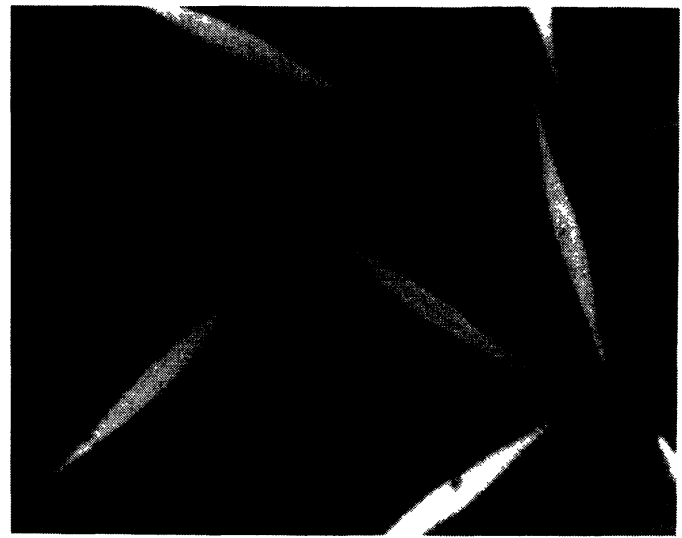

b)

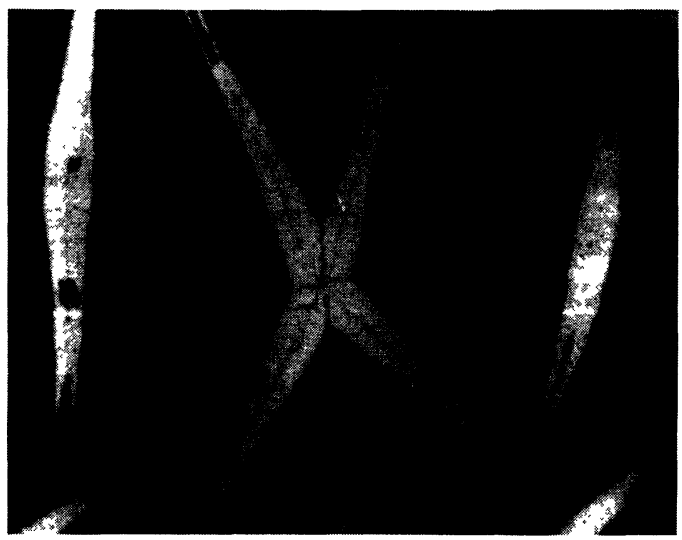

d)

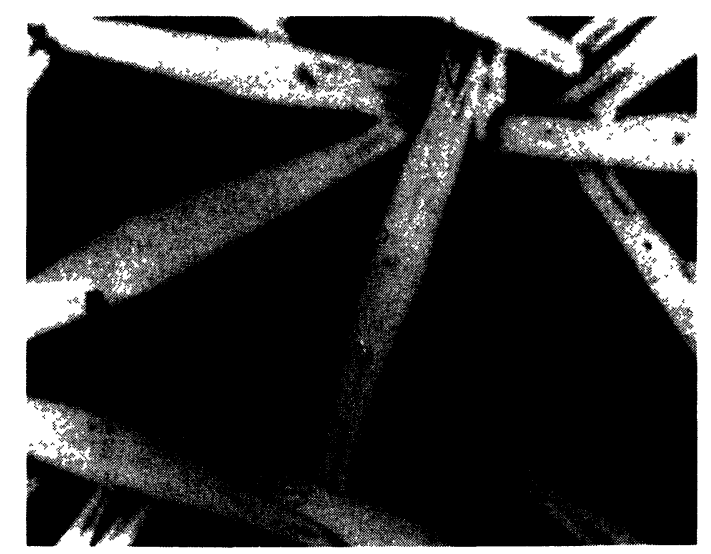

e)

Fig. 4. - Fluorescence microscopy images. The size of the pictures is $200 \times 250 \mu$. Pictures a) to e) correspond to increasing surface concentrations : a) $A=79 \AA^{2} / \mathrm{mol}$. ; b) $A=78 \AA^{2} / \mathrm{mol}$. ; c) and d) $A=70 \AA^{2} / \mathrm{mol}$. ; e) $A=65 \AA^{2} / \mathrm{mol}$. 


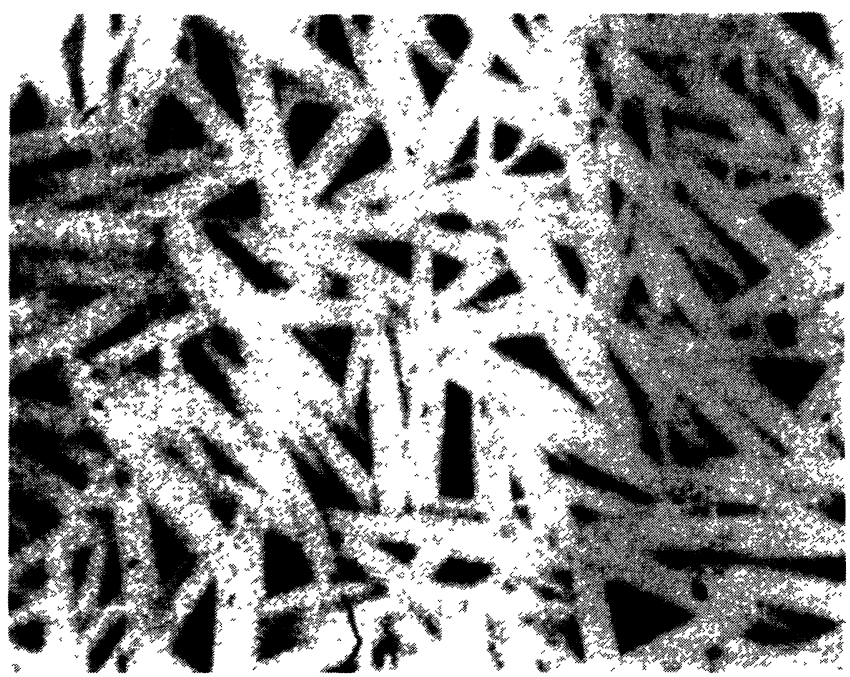

Fig. 5. - Fluorescence microscopy image of the monolayer at the largest surface concentration (before collapse) : $A=30 \AA^{2} / \mathrm{mol}$.

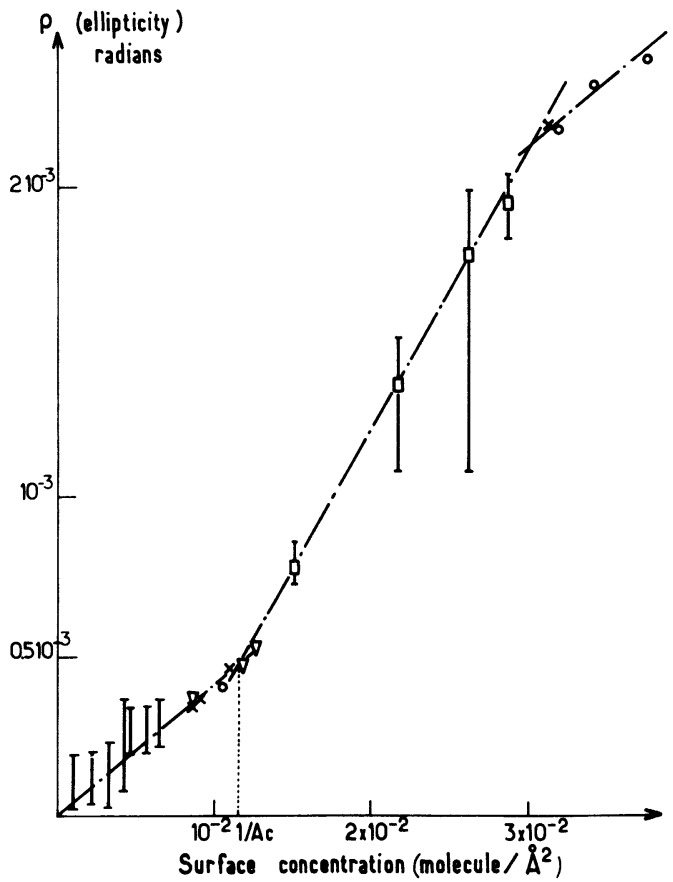

Fig. 6. - Measured ellipticity versus surface concentration. The open squares are mean values ; circles, crosses and triangles correspond to different monolayers. 
illuminated region depends upon the observation point of the surface. A second fluctuation mechanism appears in the liquid-condensed phase coexistence region : the random orientation of the anisotropic condensed domains.

The graphical representation of the mean value of $\bar{\rho}$ versus surface concentration $(\sigma=1 / A)$ is a succession of three straight lines. At low surface concentration $(\sigma<1.19 \times$ $10^{-2}$ molecule $/ \AA^{2}$ i.e. $A>84 \AA^{2} /$ molecule), no anisotropy in the monolayer plane is observed between crossed polarizer and analyzer. Supposing that the monolayer is optically isotropic, the ellipticity is given by Drude's formula. In the following, we will use a simple model where we suppose a constant density in the monolayer $\left(\rho=0.9 \mathrm{~g} / \mathrm{cm}^{3}\right)$ and a thickness d depending upon the molecular area $A: A d=M / N \rho$, where $N$ is the Avogrado number and $M$ the molecular weight. We thus obtain the refractive index in the monolayer from equation $1: n_{\mathrm{f}}=1.564$ at $\lambda=6328 \AA$.

At intermediate surface concentrations $\left(1.19 \times 10^{-2}<\sigma<3.1 \times 10^{-2}\right.$ molecule $/ \AA^{2}$ i.e. $32<A<84 \AA^{2} /$ molecule) we observe the condensation of an anisotropic two dimensional phase. The measured ellipticity is the sum of two terms as in equation (2). We will suppose that the anisotropic phase is optically uniaxial with an horizontal optical axis. Since we do not observe fluorescence in the liquid phase, and since the optical anisotropy is produced by the NBD ring, we will assume also that the corresponding dipole is inactived in the liquid phase. This leads us to take $n_{\mathrm{f}}$ as ordinary refractive index $n_{\mathrm{fo}}$ in the solid phase. In this case, the increase in slope of the curve $\bar{\rho}$ versus $\sigma$ when crossing $\sigma=1.19 \times 10^{-2}$ molecule $/ \AA^{2}$ (i.e. $\AA^{2} /$ molecule) results from the formation and the growth of the anisotropic domains. We deduce from equation (2) the value of the extraordinary refractive index $n_{\mathrm{fe}}=1.72$ (at $\lambda=6328 \AA$ and for $\rho=0.9 \mathrm{~g} / \mathrm{cm}^{3}$ ).

The average index obtained with $n_{\mathrm{fo}}=1.564$ and $n_{\mathrm{fe}}=1.72$ for randomly oriented molecules and for the same density $\rho=0.9 \mathrm{~g} / \mathrm{cm}^{3}$ is 1.61 , in good agreement with the one calculated using the additivity of the molar refraction for the different bonds present in the molecule [12]: $n_{\mathrm{f}}=1.6$ at $\lambda=5000 \AA$.

At surface concentrations larger than $3.1 \times 10^{-2}$ molecule $/ \AA^{2}$ i.e. $A<32 \AA^{2} /$ molecule, most of the monolayer is condensed in the anisotropic phase and its compression does not change the anisotropy but the thickness $d$ (or the density). The slope of the curve is approximately the same as in the liquid region.

\subsection{POLARIZED LIGHT OBSERVATIONS.}

3.4.1 Transmission. - When illuminated with blue polarized light at normal incidence, the needles whose directions are parallel to the polarization axis are not seen. This indicates that light absorption in the condensed phase is anisotropic as expected. In order to obtain quantitative information, we have illuminated the monolayer from below with a polarized beam normal to the surface, and measured the amount of transmitted light across an analyzer crossed with the polarizer.

For $A>A_{\mathrm{c}}$ the transmitted light intensity is zero. For $A<A_{\mathrm{c}}$ some of the needles are seen, i.e. those who are oriented at an angle of approximately $\pi / 4$ from the first polarizer's direction. When the analyzer is now rotated by an angle $\alpha>0$, the needles with orientations $-\pi / 4$ become brighter, those with orientations $+\pi / 4$ less bright. The contrast is maximum for $\alpha=\alpha_{0}=(30 \pm 1) 10^{-3} \mathrm{rad}$, where the needles with orientations $+\pi / 4$ are totally black (Fig. 7). These features are not affected by needles breakage.

These observations suggest that the monolayer absorption is strongly anisotropic. Let us indeed assume that the absorption is negligible when the electric field is parallel to the needle long axis and important when the field is perpendicular.

If $\varphi$ is the angle of a needle with the polarizer, the components of the electric field 


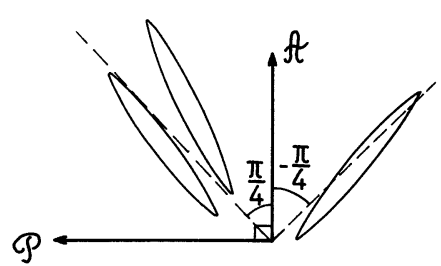

(a)

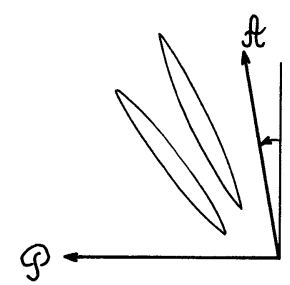

(b)

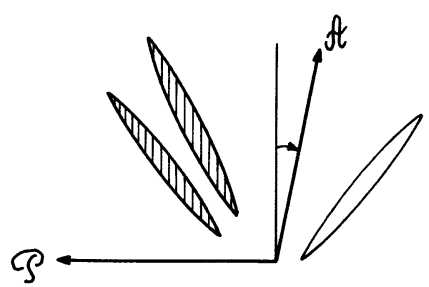

$(c)$

Fig. 7. - Schematic representation of the observations by transmission of polarized light. $P$ and $A$ are respectively the directions of the polarizer and the analyzer.

transmitted across the monolayer in the direction parallel and perpendicular to the needle are

$$
E_{\mathrm{p}}=\cos \varphi \quad E_{\mathrm{n}}=\sin \varphi \exp (-\kappa d / 2)
$$

where $\kappa$ is the absorption coefficient for the intensity. In the above expressions we have neglected the amplitude variations due to reflection.

If the analyzer is crossed with the polarizer, the transmitted field is

$$
E=\cos \varphi \sin \varphi(1-\exp (-\kappa d / 2)) .
$$

It is maximum for $|\varphi|=\pi / 4$ as observed.

If we now fix $|\varphi|=\pi / 4$, equation (3) show that the electric field is slightly rotated by passage across the monolayer. Its direction comes closer to the needle long axis by an angle $\delta$ such as

$$
\operatorname{tg} \delta=(1-\exp (-\kappa d / 2)) /(1+\exp (-\kappa d / 2))
$$

for small $\kappa d$, we get $\delta \approx \kappa d / 4$.

If the analyzer comes also closer to the needles direction $(\alpha>0 ; \varphi=-\pi / 4)$ the transmitted field increases. It becomes perpendicular to the field transmitted by other needles for which $\varphi=+\pi / 4$ when $\alpha=\delta$ (Fig. 7). The observations are thus easy to understand. Furthermore, the measurement of $\alpha_{0}$ allows to determine the product $\kappa d$ : with $\alpha_{0}=0.03$, we get $\kappa=0.12 / \mathrm{d}$. Let us compare $\kappa$ to the value that we obtain from $\kappa_{\mathrm{m}}$ for an oriented bulk phase : $\kappa^{\prime}=0.088 / \mathrm{d}$. We do not get a perfect agreement between the two measurements but the order of magnitude is correct ; furthermore, the existence of structure in the needles can modify their optical properties.

3.4.2 Reflection. - Due to the large anisotropy of its refractive index, the condensed phase may be observed by illuminating with polarized yellow light which is not absorbed by the monolayer molecules $(\lambda>5300 \AA)$ and analyzing the reflected light with an analyzer at an angle $\theta=\pi / 2$ from the polarizer. As expected the isotropic liquid phase is black. In the anisotropic phase, only the needles oriented parallel to the polarizer or analyzer directions are black. The other needles are bright. The brighter needles are oriented in the direction of the bissectrices of the polarizer and analyzer directions : $\phi= \pm 45^{\circ}$ (Fig. 8a). This shows that the neutral axes of a needle are parallel and perpendicular to this needle with corresponding indexes $n_{\mathrm{p}}$ and $n_{\mathrm{n}}$.

If the orientation of the analyzer comes closer to one type of the brighter needdles $\left(\varphi=\mp 45^{\circ}\right)$ by changing $\theta=\frac{\pi}{2}$ into $\theta=\frac{\pi}{2}+\Delta \theta$, the reflected light increases more for 


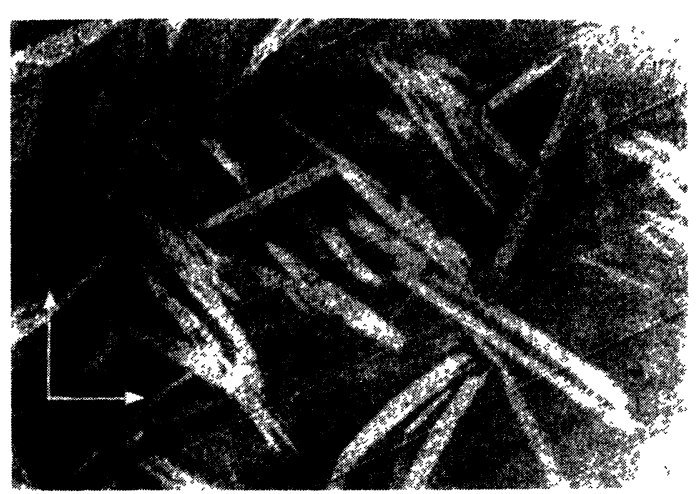

a)

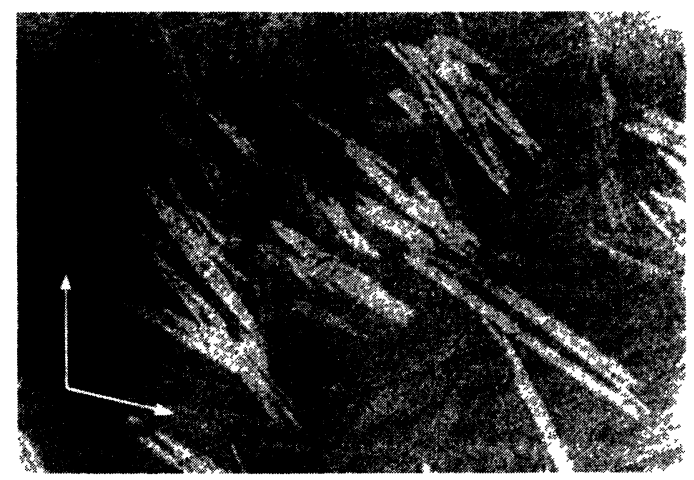

b)

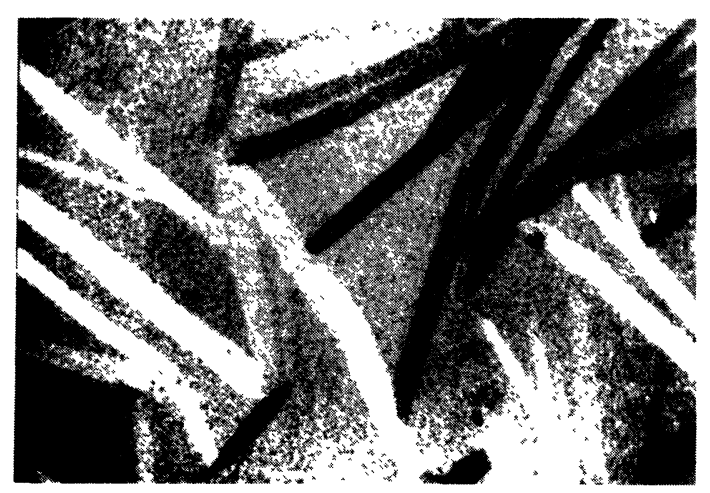

$(1)$

Fig. 8. - Microscopy images of yellow polarized light as obtained by reflection. The white arrows correspond to the directions of the polarizer and of the analyzer in a) and b). Picture c) was obtained when the residual birefringence of the microscope objective was not corrected (see text).

needles having this direction than for the perpendicular needles (Fig. $8 \mathrm{~b}$ ). This indicates that the coefficient of the linear term in $[\sin (\Delta \theta) \sin \phi]$ in the formula of the reflectivity of a needle is positive : $\left[\left(A_{\mathrm{p}}-A_{\mathrm{n}}\right)+\left(B_{\mathrm{n}}-B_{\mathrm{p}}\right)\left(B_{\mathrm{p}}+B_{\mathrm{n}}\right) / 2\right]>0$ (see appendix, Eq. (6)). Calculations shows that this is obtained if $n_{\mathrm{n}}>n_{\mathrm{p}}$. This is consistent with the assumptions made previously for the absorption by a needle (negligible when $E$ is parallel to the needle and important when $E$ is perpendicular). The intensity of the light reflected by the liquid phase around the needles is obtained by setting $A_{\mathrm{p}}=A_{\mathrm{n}}=A_{\mathrm{L}}$ and $B_{\mathrm{p}}=B_{\mathrm{n}}=B_{\mathrm{L}}$ in equation (6) of the appendix :

$$
R_{\mathrm{w}}^{2} \sin ^{2} \Delta \theta\left(1-2 A_{\mathrm{L}}+B_{\mathrm{L}}^{2}\right)
$$

where $A_{\mathrm{L}}$ and $B_{\mathrm{L}}$ are the values of $A_{\mathrm{i}}$ and $B_{\mathrm{i}}$ calculated with the refractive indices of the liquid phase $n_{\mathrm{i}}=n_{\mathrm{f}}=1.564$ and its thickness $d_{\mathrm{L}}$. One can calculate the angle $\Delta \theta$ for which the intensity reflected by a needle oriented at $\phi=45^{\circ}$ or $\phi=-45^{\circ}$ is equal to the intensity of the light reflected by the liquid phase. Using the refractive indices deduced from the ellipsometry measurements for $\lambda=6328 \AA$, i.e. $n_{\mathrm{p}}=n_{\mathrm{fo}}=1.564$ and $n_{\mathrm{n}}=n_{\mathrm{fe}}=1.72$, one finds $\Delta \theta=\mp 5.6^{\circ}$. The experimental determination of this angle $: \Delta \theta_{\exp }=6^{\circ} \pm 1^{\circ} 5$. Despite of the limited accuracy due to the low contrast, the experimental and calculated values are in agreement. 
In these observations we have taken special care that the microscope neutral axes for the objective residual birefringence were oriented parallel or perpendicular to the polarizer axis. When this is not done an asymmetry is observed for the two needles orientations $\pm 45^{\circ}$ (Fig. 8c).

Observations of reflected light between crossed polarizers is more convenient than the fluorescence methods over long periods of time : indeed we have noticed a bleaching phenomenon for NBD stearic acid in the anisotropic phase.

\section{Discussion.}

4.1 THERMODYNAMICS OF THE TRANSITION. - The transition between liquid and « solid» phases is clearly first order. The plateau below $A_{\mathrm{c}}$ is not horizontal for low $A$, but this is like for the liquid expanded-liquid condensed transition of simpler amphiphiles as discussed in the introduction.

The compression rate dependent surface pressure curves are also characteristic of a first order transition. The presence of a bump together with the microscopic observations strongly suggest that the condensed phase is formed by nucleation. The fact that the equilibrium spreading pressure $\left(\Pi_{\mathrm{e}}=29\right.$ dynes $\left./ \mathrm{cm}\right)$ is higher than the pressure $\Pi_{\mathrm{c}}=9$ dynes $/ \mathrm{cm}$ indicates that the observed phenomenon has nothing to do with the collapse.

Domains nucleation proceeds with an overpressure $\Pi-\Pi_{\mathrm{c}}$ which is an increasing function of the compression rate. The needle growth rate also increases with overpressure. A quantitative analysis of the phenomenon is in project.

4.2 ORDER IN THE PHASES. - The observations under microscope suggest that the isotropic phase is liquid-like : when some occasional draught produces convection at the surface, the needles move freely if they are far from each other. The anisotropic phase is solid-like. First, needles move through the liquid phase without any deformation. Second, when the density of needles is large, needles can be more or less bent when compressed together and eventually broken.

The observations made with polarized light show that the monolayer absorption is strongly anisotropic. The absorption is negligible when the electric field is parallel to the needle axis, and important when the field is perpendicular. The reflectivity data show that the optical axis is perpendicular to the needle ; since the molecular polarizability tensor is likely to have a large component associated to the NBD benzene ring, this suggests that the plane of the rings are oriented parallel to each other in the needle crystals.

An additional observation was made during these polarized light experiments. Any elongated part of a branched needle (see Figs. 4c-d) has the same transmission properties as a single needle (Fig. 4a). The change in dipole orientation is concentrated at the observed black lines which are then interpreted as lines of defects (dislocations) or « grain boundary » lines.

4.3 Relation tO THEORIES. - Andelman et al. [13] associated the formation of equilibrium elongated domains with long range electrostatic interactions between the molecules which either possess a charge or a vertical dipole moment. They predict a periodic organization of the expanded and condensed phases; in some cases, the phases organize in stripes whose width depends on the electrostatic parameters, although the parameters values are difficult to estimate. This theoretical (mean-field) calculation could fit with the order of magnitude of the needles width. However, in the monolayers studies here, the molecules may have also a large in-plane permanent dipole moment due to the NBD group, not taken into account in the theory. Let us note that we have only shown that they have a large optically induced dipole 
moment and that this moment may not be parallel to the permanent moment. However the molecular order evidenced might also produce an alignment of the permanent moments.

Keller et al. [14] did calculations to explain elliptic shapes and condensed phase domains separation by the competition between line tension and electrostatic energy. The width of the domains cannot be estimated, because it depends on the line tension which is unknown.

Pomeau [15] has a completely different point of view : he assumes that the domain shape is the result of growth process.

In the present work, it is difficult to decide between these different approaches. The needle shape (as observed when the pressure has reached its equilibrium value) depends on growth conditions. At this stage of the experiments it is not yet clear what are the equilibrium shapes. It is likely that the observed ones (needles) are growth shapes. Moreover, none of the theories takes into account molecular order, which seems to play an important role in the shape anisotropy.

\section{Conclusion.}

A solid anisotropic 2D phase has been evidenced, coexisting with a liquid phase in a monolayer of NBD stearic acid. Two original features of this transition makes it different from the currently investigated $2 \mathrm{D}$ phase transitions :

i) the 2D domains are straight and strongly anisotropic. They are probably not equilibrium structures and may be growth shapes;

ii) the 2D domains are optically anisotropic. The corresponding dipole moment is perpendicular to the domain long axis. Optical observations confirm the $2 \mathrm{D}$ character of these domains.

Further work is in progress to achieve a better understanding of domains shape, growth and melting.

\section{Acknowledgments.}

We have benefited from fruitful discussions with D. Chatenay, Y. Pomeau and F. Rondelez.

We gratefully acknowledge Maya Dvolaitzky who allowed us to use her Lauda film balance.

This work was supported by the Ministère de la Recherche (contrat 87C0183) and DRET (contrat 88/13444).

\section{Appendix.}

\section{Reflectivity of a needle.}

A needle is an anisotropic film of thickness $d$ with neutral axes parallel and perpendicular to the needle. Because of the anisotropy, the phase and the amplitude of the electric field $E_{\mathrm{i}}^{\mathrm{R}}$ of the light reflected on a needle depend upon the polarization [10] $\mathrm{i}$ of the incident field $E_{\mathrm{i}}$. If $\mathrm{i}=\mathrm{p}$ (incident field parallel to the needle) or if $\mathrm{i}=\mathrm{n}$ (incident field perpendicular to the needle) one obtains :

$$
E_{\mathrm{i}}^{\mathrm{R}}=R_{\mathrm{i}} E \quad \text { with }: \quad R_{\mathrm{i}}=\frac{r_{01}+r_{12} \exp i \beta}{1+r_{01} r_{12} \exp i \beta}
$$

and :

$$
r_{01}=\frac{n_{\mathrm{i}}-1}{n_{\mathrm{i}}+1} ; \quad r_{12}=\frac{n_{\mathrm{w}}-n_{\mathrm{i}}}{n_{\mathrm{w}}+n_{\mathrm{i}}} ; \quad \beta=4 \pi n_{\mathrm{i}} \frac{d}{\lambda}
$$

$n_{\mathrm{i}}=n_{\mathrm{p}}$ or $n_{\mathrm{i}}=n_{\mathrm{n}}$ with a polarization parallel $(\mathrm{i}=\mathrm{p})$ or perpendicular $(\mathrm{i}=\mathrm{n})$ to the needle. 
If $\phi$ is as before, the angle between the polarizer and the needle, $\theta=\frac{\pi}{2}+\Delta \theta$, the angle of the analyzer with the polarizer, the component parallel to the analyzer of the reflected electric field is :

$$
\left\{R_{\mathrm{p}} \cos \varphi \sin (\varphi+\Delta \theta)-R_{\mathrm{n}} \sin \varphi \cos (\varphi-\Delta \theta)\right\} E .
$$

Up to second order in $d / \lambda$, the reflectivity of a needle is :

$$
\begin{aligned}
& R_{\mathrm{w}}^{2}\left\{\sin ^{2} \Delta \theta\left[1-2\left(A_{\mathrm{p}} \cos ^{2} \varphi+A_{\mathrm{n}} \sin ^{2} \varphi\right)+\left(B_{\mathrm{p}} \cos ^{2} \varphi+B_{\mathrm{n}} \sin ^{2} \varphi\right)^{2}\right]+\right. \\
& \quad+\left(B_{\mathrm{p}}-B_{\mathrm{n}}\right)^{2} \cos ^{2} \varphi \sin ^{2} \varphi \cos ^{2} \Delta \theta \\
& \left.\quad+2 \sin \Delta \theta \cos \Delta \theta \sin \varphi \cos \varphi\left[\left(A_{\mathrm{p}}-A_{\mathrm{n}}\right)+\left(B_{\mathrm{n}}-B_{\mathrm{p}}\right)\left(B_{\mathrm{p}} \cos ^{2} \varphi+B_{\mathrm{n}} \sin ^{2} \varphi\right)\right]\right\}
\end{aligned}
$$

where :

$$
\begin{aligned}
& A_{\mathrm{i}}=4 \pi \frac{d}{\lambda} \frac{n_{\mathrm{w}}^{2}-n_{\mathrm{i}}^{2}}{n_{\mathrm{w}}^{2}-1} \\
& B_{\mathrm{i}}=\left(4 \pi \frac{d}{\lambda}\right)^{2} \frac{n_{\mathrm{i}} V_{\mathrm{i}}}{2}\left[1-\frac{\left(n_{\mathrm{w}}-n_{\mathrm{i}}\right)\left(n_{\mathrm{i}}-1\right)}{n_{\mathrm{i}}^{2}\left(n_{\mathrm{w}}+1\right)} U_{\mathrm{i}}\right] \\
& R_{\mathrm{w}}=\frac{n_{\mathrm{w}}-1}{n_{\mathrm{w}}+1}
\end{aligned}
$$

\section{References}

[1] GaINEs G. L., Insoluble monolayers at liquid-gas interface (Wiley, New-York) 1966.

[2] Bell G. M., Combs L. L. and Dunne L. J., Chem. Rev. 81 (1981) 15 ;

Legre J. P., Albinet G., Firpo J. L. and Tremblay A. M. S., Phys. Rev. A 30 (1984) 2720 and references therein.

[3] Middleton S. R., Iwasaki M., Pallas N. R. and Pethica B. A., Proc. R. Soc. London Ser. A 396 (1984) 143 ;

Pallas N. R. and Pethica B. A., Langmuir 1 (1985) 509.

[4] Losche M., Sackmann E. and Mohwald H., Ber. Bunsenges Phys. Chem. 87 (1983) 848.

[5] McConnell H. M., Tann L. K. and Weis R. M., Proc. Natl. Acad. Sci. USA 81 (1984) 3249 ;

Weiss R. M. and McConNell H. M., Nature 310 (1984) 47 ;

Gaub H. E., Moy V. T. and McConnell H. M., J. Phys. Chem. 90 (1986) 1721.

[6] Moore B., Knobler Ch. M., Broseta D. and Rondelez F., J. Chem. Soc., Far. Trans. 282 (1986) 1753 ;

Rondelez F. and Suresh K. A., Physics of Amphiphilic layers, Eds. J. Meunier, D. Langevin and N. Boccara (Springer) 1987.

[7] Losche M., Duwe H. P. and Mohwald, J. Coll. Int. Sci., in press.

[8] Jasperson S. N., Schnatterly S. E., Rev. Sci. Instrum. 40 (1969) 761.

[9] Drude P., Lehrbuch des Physiks, Berlin (1899).

[10] Azzam R. M. A. and Bashara N. M., Ellipsometry and polarized light (North Holland) 1977.

[11] Rasing Th., Hsiung H., Shen Y. R. and Kim M. W., Phys. Rev. A 37 (1988) 2732.

[12] Handbook of Chem. and Phys., 44th edition, Ed. Ch. D. Hodgman (Cleveland) 1962.

[13] Andelman D., Brochard F. and Joanny J. F., J. Chem. Phys. 86 (1986) 3673.

[14] Keller D. J., Korb J. P. and McConnell H. M., J. Phys. Chem. 91 (1987) 6417.

[15] PomeaU Y., Europhys. Lett. 3 (1987) 1201. 